\title{
Claudia ZeY
}

\section{AUTOUR DES SOULÈVEMENTS COMMUNAUX EN ITALIE SEPTENTRIONALE ET CENTRALE AUX XI ${ }^{\mathrm{e}}$ ET XII ${ }^{\mathrm{e}}$ SIÈCLES}

En Italie, le mouvement communal prit son origine comme en France, dans le nord du pays ${ }^{1}$. On rencontre les premiers soulèvements communaux dans la région lombarde au milieu du $\mathrm{XI}^{\mathrm{e}}$ siècle, et ils atteignirent les villes toscanes vers les alentours de 1100 . Rome ne fut touchée par le mouvement communal que vers le milieu du XII ${ }^{e}$ siècle $^{2}$, tandis que l'Italie méridionale passa au cours des $\mathrm{XI}^{\mathrm{e}}$ et $\mathrm{XII}^{\mathrm{e}}$ siècles sous la domination plus centralisatrice des Normands qui surent presque toujours opprimer les penchants des villes vers l'autonomie'.

La Lombardie comme noyau d'éclosion du mouvement communal fut jugée digne d'une explication assez développée par Otton de Freising quand celui-ci dressa un portrait littéraire de son neveu Frédéric Barberousse et de ses actions ${ }^{4}$. Otton interrompit le récit des événements de la première expédition du roi de la dynastie des Staufen et futur empereur vers l'Italie en l'an 1154 au profit d'un portrait de la civilisation italienne en général et de la Lombardie en particulier. Après des indications portant sur la géographie et la toponymie, Otton se penche sur la situation politique.

Dans l'administration des villes également et dans le souci du maintien de l'ordre politique, les habitants de l'Italie prennent l'art politique des vieux Romains comme modèle. Mais surtout ils éprouvent un tel amour de la liberté que dans le désir de se soustraire à un exer-

Je remercie M. Julian Führer pour la traduction de cette contribution en français.

${ }^{1}$ Le concept géographique d'Italie septentrionale et centrale correspond à l'Italie impériale (regnum Italie), or cette désignation évoquerait un antagonisme entre les villes et le pouvoir royal lors du début de la phase communale qui n'existait pas au degré qu'on constate du temps de Frédéric Barberousse. Voir p.ex. les développements de Knut SCHULz, «Denn sie lieben die Freiheit so sehr...«. Kommunale Aufstände und Entstehung des europäischen Bürgertums im Hochmittelalter, Darmstadt 1992, p. 212-214 sur l'action des représentants de la ligue lombarde pour honor et libertas Italie d'après la description de Romuald de Salerne.

${ }^{2}$ Le développement communal de Rome ne fait pas partie des centres d'intérêt de cet article.

${ }^{3}$ Voir SCHULZ, "Denn sie lieben die Freiheit so sehr« (voir n. 1), p. 7.

${ }^{4}$ Sur les Gesta Friderici voir en dernier lieu Lars HAGENEIER, Die frühen Staufer bei Otto von Freising oder Wie sind die Gesta Friderici entstanden?, dans: Hubertus SEIBERT, Jürgen DENDORFER (dir.), Grafen, Herzöge, Könige. Der Aufstieg der frühen Staufer und das Reich (1079-1152), Ostfildem 2005 (Mittelalter-Forschungen, 18), p. 363-396. 
cice arbitraire du pouvoir, ils préfèrent se soumettre à l'arbitrage de consuls qu'à la volonté ordinatrice de souverains. Comme est bien connu qu'ils connaissent trois ordres sociaux, à savoir les capitanei, les vavasseurs et le menu peuple, on choisit ces consuls non pas parmi un seul, mais parmi tous les trois états afin d'éviter tout orgueil; et pour que ceux-ci ne prennent pas le goût de la domination, on les remplace presque tous les ans. Ainsi, comme tout le pays est divisé en cités, chaque ville a obligé les habitants de son diocèse à s'adjoindre à elle, et on ne trouvera guère de noble ou puissant de telle envergure qu'il ne donne suite aux prescriptions de sa cité. Grâce à ce pouvoir de contrainte, les villes en question ont l'habitude de désigner les territoires respectifs comme leursı comtés. Afin de ne pas manquer des moyens de tenir en échec leurs voisins, ils n'hésitent pas à admettre à la dignité de chevaliers de jeunes hommes de basse condition et même des artisans s'adonnant à quelque vil métier mécanique et que d'autres peuples écartent comme la peste des tâches honorables et plus dignes des libres, et à leur permettre d'accéder à de plus hautes fonctions. Ainsi les villes lombardes dépassent les autres villes du monde en richesse et en puissance ${ }^{5}$.

Cette description des communes de l'Italie septentrionale dégageant l'admiration et l'horreur à la fois ${ }^{6}$ provient d'un représentant éminent du "monde

${ }^{5}$ Otto von Freising (und Rahewin), Gesta Friderici I. imperatoris II 13, éd. par Georg WAITZ, Bernhard VON SIMSON, Hanovre, Leipzig 1912 (MGH SS rer. Germ., [46]), S. 116: In civitatum quoque dispositione ac rei publicae conservatione antiquorum adhuc Romanorum imitantur sollertiam. Denique libertatem tantopere affectant, ut potestatis insolentiam fugiendo consulum potius quam imperantium regantur arbitrio. Cumque tres inter eos ordines, id est capitaneorum, vavassorum, plebis, esse noscantur, ad reprimendam superbiam non de uno, sed de singulis predicti consules eliguntur, neve ad dominandi libidinem prorumpant, singulis pene annis variantur. Ex quo fit, ut, tota illa terra inter civitates ferme divisa, singulae ad commanendum secum diocesanos compulerint, vixque aliquis nobilis vel vir magnus tam magno ambitu inveniri queat, qui civitatis suae non sequatur imperium. Consueverunt autem singuli singula territoria ex hac comminandi potestate comitatus suos appellare. Ut etiam ad comprimendos vicinos materia non careant, inferioris conditionis iuvenes vel quoslibet contemptibilium etiam mechanicarum artium opifices, quos caeterae gentes ab honestioribus et liberioribus studiis tamquam pestem propellunt, ad miliciae cingulum vel dignitatum gradus assumere non dedignantur. Ex quo factum est, ut caeteris orbis civitatibus divitiis et potentia [longe] premineant. La traduction en langue française suit la traduction en allemand par Hagen KELLER, Adelsherrschaft und städtische Gesellschaft in Oberitalien 9. bis 12. Jahrhundert, Tübingen 1979 (Bibliothek des Deutschen Historischen Instituts in Rom, 52), p. 17-18, n. 1. La traduction allemande par Adolf SCHMIDT, Bischof Otto von Freising und Rahewin, Die Taten Friedrichs oder richtiger Cronica, dir. par FranzJosef SCHMALE, Darmstadt 1965 (Ausgewählte Quellen zur deutschen Geschichte des Mittelalters. Freiherr vom Stein-Gedächtnisausgabe, 17), p. 309-310 (ici II 14) pose problème à cause d'anachronismes dans la terminologie. Les expressions d'Otton de Freising ressemblent de près à la caractérisation que donne André de Strumi dans sa Vita Sancti Arialdi c. 2, éd. par Friedrich BAETHGEN, dans: MGH SS 30, 2, Leipzig 1934, p. 1047-1075, ici p. 1050: Porro superbiam, quae est causa diaboli, ita execrabantur, ut, cum libere modis omnibus suis vicinis imminerent atque a nemine eorum, si nollent, constringi possent, ceu essent ex illorum minimis, sic se ultro subdebant ipsorum omni iustae conventioni.

${ }^{6}$ Voir l'explication détaillée du texte chez KELLER, Adelsherrschaft und städtische Gesellschaft (voir n. 5), p. 18-21. Sur le »mélange entre étonnement, effroi et répulsion envers les soulevements communaux « (»Mischung aus Erstaunen, Entsetzen und Abscheu auf die kommunalen Erhebungen«) chez toute une série d'auteurs contemporains voir SCHULZ, 
aristocratique de la domination au Moyen Âge« ${ }^{7}$ qui dresse un tableau simpliste des communes comme des collectivités citadines par contrainte. Il reconnaît aux communes un caractère répressif vers l'intérieur comme vers l'extérieur et les caractérise comme permissives dans leur structure sociale. Par cette description simplifiée Otton de Freising cherche à discréditer leurs revendications vers l'autodétermination politique et à souligner en même temps la légalité des prétentions royales et impériales à la domination des villes dans le règne d'Italie.

Malgré cette façon tendancieuse de présenter la situation, les développements du célèbre chroniqueur de Freising constituent un fil directeur approprié pour l'étude du thème "Révolte et statut social« dans l'espace citadin de l'Italie du nord. Otton de Freising traite l'ordre social des communes en plusieurs endroits. La répartition dans les trois conditions supérieures des capitanei, vavasseurs et citadins est présumée par lui comme connue au milieu du $\mathrm{XII}^{\mathrm{e}}$ siècle $^{8}$. La participation des classes inférieures et des artisans à l'administration des villes constitue pour lui le trait hors du commun qui apporte aux communes de l'Italie du nord la richesse et la puissance qui les fait devancer toutes les autres villes du monde. Il sera donc question ici des débuts de ce développement qui a mené à l'intégration de toutes les classes sociales dans la constitution communale. Il se pose plus concrètement la question de savoir si et dans quelle mesure des représentants de tous les états ont participé aux soulèvements communaux du $\mathrm{XI}^{\mathrm{e}}$ siècle. Dans un second temps il faudra chercher les premiers indices pour l'action des forces respectives menant à l'association de classes et groupes dans une constitution communale qui selon Otton de Freising a forcé même les nobles à la vie au sein d'une commune. Dans une troisième partie, enfin, sera éclaircie la question de la perception et de l'appréciation des mouvements communaux et de leurs meneurs dans

»Denn sie lieben die Freiheit so sehr« (voir n. 1), p. 14-15, 196-197 sur le passage cité d'Otton de Freising.

${ }^{7}$ Gerhard DILCHER, Mittelalterliche Stadtkommune, Städtebünde und Staatsbildung. Ein Vergleich Oberitalien - Deutschland, dans: Heiner LÜCK, Bernd SCHILDT (dir.), Recht Idee - Geschichte. Beiträge zur Rechts- und Ideengeschichte für Rolf Lieberwirth anläßlich seines 80. Geburtstages, Cologne, Weimar, Vienne 2000, p. 453-467, ici p. 459: waristokratischen Herrschaftswelt des Hochmittelaiters«. Voir également Hans-Werner GOETZ, Das Geschichtsbild Ottos von Freising. Ein Beitrag zur historischen Vorstellungswelt und zur Geschichte des 12. Jahrhunderts, Cologne, Vienne 1984 (Beihefte zum Archiv für Kulturgeschichte, 19), p. 27.

${ }^{8}$ Sur l'évolution de la terminologie des couches sociales, voir l'ouvrage fondamental de KELLER, Adelsherrschaft und städtische Gesellschaft (voir n. 5), p. 36-60 ainsi que, pour l'usage de la terminologie du mouvement communal chez les chroniqueurs principaux des $\mathrm{XI}^{e}$ et $\mathrm{XII}^{e}$ siècles, ID., Die soziale und politische Verfassung Mailands in den Anfängen des kommunalen Lebens. $\mathrm{Zu}$ einem neuen Buch über die Entstehung der lombardischen Stadtkommune, dans: Historische Zeitschrift 211 (1970), p. 34-64, ici p. 41-49. 
l'historiographie des $\mathrm{XI}^{\mathfrak{e}}$ et $\mathrm{XII}^{\mathrm{e}}$ siècles comme cela est fourni par Otton de Freising.

Dans le cadre restreint de cette étude, on ne pourra fournir que des éclairages partiels; surtout, il ne sera pas possible de différencier toujours entre les différentes villes. Des considérations d'ordre plus général sont néanmoins permises, étant donné que le paysage citadin de l'Italie septentrionale et centrale est marqué par grand nombre de points communs qui le distingue des villes dans d'autres régions de l'Europe, à commencer par la continuité ininterrompue de l'évolution urbaine en Italie depuis l'Antiquité. La prédominance de la vie urbaine continua de marquer la structure sociale de la région même après la chute de l'Empire romain et les désordres des invasions barbares ${ }^{9}$. La noblesse, classe dirigeante à l'époque des Carolingiens, des Ottoniens et des Saliens, continuait à résider dans les villes. Nombre de vassaux royaux y habitaient ou du moins y possédaient des biens étendus. Eux-mêmes faisaient partie de la classe dirigeante dans les villes ${ }^{10}$. La noblesse urbaine profita, pour développer son pouvoir, du fait que l'autorité royale ne s'imposa jamais au même degré en Italie que dans le royaume franc oriental. Les nombreuses expéditions et les longs séjours des souverains ottoniens au $\mathrm{X}^{\mathrm{e}}$ siècle $\mathrm{n}$ ' $\mathrm{y}$ changèrent rien. Les rois renonçaient au contraire à certains instruments du pouvoir comme les droits fiscaux et la souveraineté justicière au profit des évêques, sans toutefois pouvoir disposer régulièrement de l'occupation des sièges épiscopaux comme c'était le cas au nord des Alpes ${ }^{11}$. Le pouvoir des évêques seigneurs des villes et des familles nobles où se recrutait l'épiscopat fut contré avec succès par le reste de la classe supérieure de la ville grâce à sa lutte pour l'hérédité des droits d'usufruit et d'administration qu'elle avait reçus en fief de possessions impériales et ecclésiastiques par l'évêque. L'hérédité de ces fiefs fut garantie par la grande loi féodale de Conrad II, la constitutio de feudis de l'an $1037^{12}$.

${ }^{9}$ Voir Gerhard DILCHER, Bischof und Stadtverfassung in Oberitalien, dans: Zeitschrift der Savigny-Stiftung für Rechtsgeschichte, Germanistische Abteilung 81 (1964), p. 225-266.

${ }^{10}$ Voir Hagen KELLER, Der Übergang zur Kommune: Zur Entwicklung der italienischen Stadtverfassung im 11. Jahrhundert, dans: Bernhard DiESTELKAMP (dir.), Beiträge zum hochmittelalterlichen Städtewesen, Cologne, Vienne 1982 (Städteforschung, Reihe A: Darstellungen, 11), p. 55-72, surtout p. 56-57.

${ }^{11}$ Voir DILCHER, Bischof und Stadtverfassung (voir n. 9), p. 235-250; ID., Die Entstehung der lombardischen Stadtkommune, Aalen 1967 (Untersuchungen zur deutschen Staats- und Rechtsgeschichte. Neue Folge, 7), p. 88-98; KELLER, Der Übergang zur Kommune (voir n. 10 ), p. $57-59$.

${ }^{12}$ Voir Die Urkunden Konrads II. mit Nachträgen zu den Urkunden Heinrichs II., éd. par Harry BRESSLAU, Hanovre, Leipzig 1909 (MGH Diplomata regum et imperatorum, 4), $\mathrm{n}^{\circ} 244$ (DKo. II. 244); voir à ce sujet KELLER, Adelsherrschaft und städtische Gesellschaft (voir n. 5), p. 286-291 et en dernier lieu, après KELLER, Herwig WOLFRAM, Konrad II., 990-1039. Kaiser dreier Reiche, Munich 2000, p. 147-148. 
Les liens étroits entre l'Église et la noblesse urbaine ne concernaient toutefois pas que l'évêque avec sa famille et sa suite féodale, mais aussi le haut clergé. Dans la mesure où l'on est renseigné sur l'origine des membres du clergé cathédral, les constats sont plutôt clairs. Les membres des chapitres cathédraux étaient originaires en majorité des familles de la noblesse urbaine ${ }^{13}$. Prenant en considération les relations étroites des classes dirigeantes ecclésiastique et séculière, il n'est guère surprenant que des troubles sociaux aient nécessairement perturbé la vie spirituelle et que des revendications dans le sens de la réforme de l'Église aient affecté la structure sociale des villes italiennes dans son ensemble. Inversement, on pourra s'étonner du fait que les meneurs des insurrections motivées aussi bien socialement que spirituellement dans les communes urbaines d'Italie venaient de familles nobles. L'observation selon laquelle les chefs de mouvements révolutionnaires ne provenaient pas d'une manière générale de la même couche sociale que la masse de leurs partisans et de ceux qui les soutenaient ne constitue pas un fait nouveau ou limité au Moyen Âge ${ }^{14}$. Pour le phénomène des révoltes urbaines, ce fait restera néanmoins plus remarquable que dans un contexte rural, car le statut de liberté juridique n'était pas limité aux nobles dans les villes ${ }^{15}$ et ceux-ci ne disposaient pas - du moins en Italie - du monopole sur l'éducation et la culture ${ }^{16}$. Pour ce qui est du prestige social en ville, les riches marchands n'étaient en rien inférieurs aux vassaux nobles ${ }^{17}$. Pourtant, lors de révoltes communales on les dis-

${ }^{13}$ Hagen KeLLER, Origine sociale e formazione del clero cattedrale dei secoli XI e XII nella Germania e nell'Italia settentrionale, dans: Le istituzioni ecclesiastiche della >societas christianar dei secoli XI-XII. Diocesi, pievi e parrocchie. Atti della sesta settimana internazionale di studio, Milano, 1-7 settembre 1974, Milan 1977 (Pubblicazioni dell'Università Cattolica del Sacro Cuore. Miscellanea del Centro di Studi Medioevali, 8), p. 136-186, surtout p. 144-159 ainsi que les trois annexes p. 160-186. Pour Milan, voir ID., Die soziale und politische Verfassung Mailands (voir n. 8), p. 36-38 ainsi que l'annexe p. 62-63; ID., Adelsherrschaft und städtische Gesellschaft (voir n. 5), p. 224-227, 397-398. Pour Plaisance, voir Pierre RACINE, La nascita del comune, in: Storia di Piacenza, Bd. 2: Dal vescovo conte alla signoria (996-1313), Plaisance 1984, p. 49-74, surtout p. 63, 65; Olaf ZUMHAGEN, Religiöse Konflikte und kommunale Entwicklung. Mailand, Cremona, Piacenza und Florenz zur Zeit der Pataria, Cologne, Weimar, Vienne 2002 (Städteforschung, Reihe A: Darstellungen, 58), p. 157. ${ }^{14}$ Voir p.ex. Wolfgang vON WARTBURG, Sie haben die Welt verändert: Revolutionäre Gestalten des 19. und 20. Jahrhunderts, Schaffhausen ${ }^{2} 1974$; Anja Victorine HaRTMANN, Kontinuitäten oder revolutionärer Bruch? Eliten im Übergang vom Ancien Régime zur Moderne. Eine Standortbestimmung, dans: Zeitschrift für historische Forschung 25 (1985), p. 389-420.

${ }^{15}$ Voir KELLER, Der Übergang zur Kommune (voir n. 10), p. 56-57.

${ }^{16}$ Voir p.ex. les remarques de Jörg W. BUSCH, Die Mailänder Geschichtsschreibung zwischen Arnulf und Galvaneus Flamma. Die Beschäftigung mit der Vergangenheit im Umfeld einer oberitalienischen Kommune vom späten 11. bis zum frühen 14. Jahrhundert, Munich 1997 (Münstersche Mittelalter-Schriften, 72), p. 44.

17 Voir KeLLER, Adelsherrschaft und städtische Gesellschaft (voir n. 5), p. 25, 34, 45-46, 249-250, 398-399. 
tingue plutôt au deuxième rang, et non comme des acteurs de premier plan agissant au grand jour ${ }^{18}$.

Les sources pertinentes des $\mathrm{XI}^{\mathrm{e}}$ et $\mathrm{XII}^{\mathrm{e}}$ siècles traduisent une conscience marquée chez les contemporains de l'appartenance de chaque personne à une classe sociale ${ }^{19}$. Les nobles ne sont que rarement désignés par les termes généraux de nobiles ou nobilitas, plus souvent et bien avant 1100 furent utilisés les termes plus différenciés qu'emploie aussi Otton de Freising au milieu du $\mathrm{XII} \mathrm{I}^{\mathrm{e}}$ siècle. En premier lieu les capitanei, les grands feudataires des rois, margraves, comtes et évêques. Les petits feudataires, les vavasseurs (ou vasvassores) leur sont subordonnés et dépendent d'eux. Le reste non noble de la population est ou bien désigné indifféremment par plebs et populus ou bien on établit une autre distinction plus fine comme dans un acte expédié en 1067 pour la ville de Milan ${ }^{20}$ où figurent les marchands, les negotiatores, et les autres habitants, reliqui ou alii cives. Au milieu du $\mathrm{XI}^{\mathrm{e}}$ siècle, seuls les ressortissants des familles des capitanei sont désignés comme chevaliers, milites. Avec le rapprochement de la noblesse des grands et petits feudataires vers le tour-

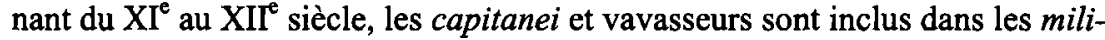
tes. $\mathrm{Au} \mathrm{XII}{ }^{\mathrm{e}}$ siècle, enfin, on peut trouver des chevaliers qui, sans être nobles, combattent à cheval et portent les armes.

La conscience de l'appartenance de chacun à un état social était d'une importance particulière chez les meneurs de révoltes urbaines. Leur origine sociale était connue de toute la ville et nous est mentionnée par les chroniqueurs commentant les événements. Quand en 1042 le peuple de Milan se révolta contre le joug oppressant de la noblesse urbaine, il fut dirigé par un miles Lan$z o^{21}$. Pendant des décennies avant et après cette révolte, Lanzo exerça l'office de

${ }^{18}$ Pour les monnayeurs et marchands dans l'entourage de la Pataria milanaise, voir Hagen KELLER, Pataria und Stadtverfassung, Stadtgemeinde und Reform: Mailand im »Investiturstreit«, dans: Josef FLECKENSTEN (dir.), Investiturstreit und Reichsverfassung, Sigmaringen 1973 (Vorträge und Forschungen, 17), p. 321-350, ici p. 328; Paolo GoLINELLI, La pataria. Lotte religiose e sociali nella Milano dell'XI secolo, Milan 1984, p. 42-45.

${ }^{19}$ Pour ce qui suit, voir KELLER, Adelsherrschaft und städtische Gesellschaft (voir n. 5), p. 25-36 avec les références ainsi que les passages mentionnés plus haut à la note 8 et ID., Mailand im 11. Jahrhundert. Das Exemplarische an einem Sonderfall, dans: Jörg JARNUT, Peter JOHANEK (dir.), Die europäische Stadt im 11. Jahrhundert, Cologne, Weimar, Vienne 1998 (Städteforschung, Reihe A: Darstellungen, 43), p. 81-104, ici p. 98-104.

${ }^{20}$ Il s'agit ici d'un acte de deux légats pontificaux (le cardinal-évêque Mainard de Silva Candida et le cardinal-prêtre Jean Minutus de S. Maria in Trastevere), passé vers la fin de leur légation à Milan vers la fin du mois de juillet 1067 pour mettre un terme aux attaques des partisans de la Pataria contre le clergé milanais. Voir l'édition de l'acte d'après plusieurs rédactions chez Joannes Dominicus MANSI, Sacrorum conciliorum nova et amplissima collectio. Editio novissima 19, Paris 1902, col. 946-948; Julius vON PFLUGK-HARTTUNG, Iter Italicum, Stuttgart 1883, p. $424-429, n^{\circ} 39$.

${ }^{21}$ Pour le soulèvement populaire milanais de 1042-1044, voir Arnulf von Mailand, Liber gestorum recentium II 18-19, éd. par Claudia ZEY, Hanovre 1994 (MGH SS rer. Germ., 67), p. 163-165; Landulfi Historia Mediolanensis II 26-28, éd. par Ludwig Konrad BETHMANN, 
juge royal à Milan, et remplit temporairement la fonction de messager royal ${ }^{22} . \AA$ ses côtés se trouve un homme dont le prénom, Alberius, est assez fréquent dans les milieux marchands, plus tard dans les familles de vavasseurs ${ }^{23}$.

Le même modèle social est connu pour les dirigeants du mouvement de réforme de l'Église que ses opposants appelaient dans uns sens péjoratif »Pataria $\varkappa^{24}$. On désignait ainsi ceux qui soutenaient énergiquement, et parfois en usant de violence, la réalisation des revendications de réforme au sein de l'Église et en particulier l'imposition du célibat sacerdotal et l'interdiction de l'achat des offices ecclésiastiques. La première phase de son action à Milan entre 1056 et 1075 est liée aux noms des clercs Ariald et Landulf et à celui de son frère, le chevalier Erlembald ${ }^{25}$. Alors que la famille d'Ariald, originaire de la région de Côme, peut être classée parmi les vavasseurs ${ }^{26}$, Landulf et Erlembald faisaient partie d'une famille de capitanei milanais. Landulf était même membre du clergé cathédral de Milan ${ }^{27}$. Dans le proche entourage de ce groupe dirigeant on arrive encore à identifier quelques monetarii. Ces mon-

Wilhelm WATTENBACH, dans: MGH SS 8, Hanovre 1848 , p. $32-100$, ici p. $62-67 /$ Landulfi senioris Mediolanensis Historiae libri quattuor II 26-28, éd. par Alessandro CUTOLO, Bologne 1934 (Rerum Italicarum Scriptores, ${ }^{24}$, 2), p. 63-70. Sur les deux éditions de Landulf voir en dernier lieu Paolo CHIESA, "Landolfo Seniore«, dans: Dizionario biografico degli Italiani 63 (2004), p. 497-501, ici p. 500.

${ }^{22}$ Les références se trouvent chez KELLER, Die soziale und politische Verfassung Mailands (voir n. 8), p. 37 n. 16; ID., Adelsherrschaft und städtische Gesellschaft (voir n. 5), p. 209-210.

${ }^{23}$ Voir Landulf l'Ancien, Historia Mediolanensis (voir n. 21) II 26, MGH SS 8, p. 64/RIS ${ }^{24}$, 2, p. 65. Pour l'identification des noms de personnes, voir KELLER, Adelsherrschaft und städtische Gesellschaft (voir n. 5), p. 237-241.

${ }^{24}$ Voir Cinzio VIOLANTE, La pataria milanese e la riforma ecclesiastica 1: Le premesse (1045-1057), Rome 1955 (Studi storici, 11-13); ID., I laici nel movimento patarino, dans: I laici nella >societas christiana dei secoli XI e XII. Atti della terza settimana internazionale di studio, Mendola, 21-27 agosto 1965, Milan 1968 (Miscellanea del Centro di studi medioevali, 5), p. 597-687 (repris dans: ID., Studi sulla christianità medioevale. Società - Istituzioni Spiritualità, raccolti da Piero ZERBI, Milan 1972 [Cultura e storia, 8], p. 145-246); KELLER, Pataria und Stadtverfassung (voir n. 18), avec le relevé de la bibliographie plus ancienne p. 325, n. 9; Helmut GRITSCH, Die Pataria von Mailand (1057-1075), dans: Innsbrucker Historische Studien 3 (1980), p. 7-42; GoLINELLI, La pataria (voir n. 18); Hagen KELLER, Olaf ZUMHAGEN, "Pataria«, in: Theologische Realenzyklopädie 26 (1996), p. 83-85; KELLER, Mailand im 11. Jahrhundert (voir n. 19); ZUMHAGEN, Religiöse Konflikte und kommunale Entwicklung (voir n. 13).

${ }^{25}$ À côté des auteurs milanais Amulf et le soi-disant Landulf l'Ancien, hostiles à la Pataria, l'abbé André du monastère réformateur de Strumi du courant de Vallombreuse a décrit l'action de la Pataria d'une façon détaillée dans une perspective favorable dans sa Vita Sancti Arialdi (voir n. 5), de même le cardinal-évêque Bonizon de Sutri, plus tard évêque de Plaisance dans son Liber ad amicum VI et VII, éd. par Ernst DÜMMLER, dans: MGH Libelli de lite 1, Hanovre 1891, p. 590-609. Voir les brefs aperçus chez GolinelLI, La pataria (voir n. 18), p. 23-33; KELLER, Mailand im 11. Jahrhundert (voir n. 19), p. 94.

${ }^{26}$ Voir ID., Adelsherrschaft und städtische Gesellschaft (voir n. 5), p. 166.

${ }^{27}$ Voir ID., Origine sociale e formazione del clero cattedrale (voir n. 13), p. 185-186; ID., Adelsherrschaft und städtische Gesellschaft (voir n. 5), p. 26, 210-212. 
nayeurs ou changeurs de monnaie appuyaient durablement les partisans de la Pataria avec des moyens financiers et leur fournissaient même des maisons afin qu'ils y réalisent la vita communis qu'ils désiraient mener ${ }^{28}$. On connaît également des bienfaiteurs et partisans de la Pataria dans les milieux professionnels ambitieux des artisans et marchands que le sentiment de leur propre valeur encourageait apparemment à vouloir participer aux prises de décision ecclésiastiques ${ }^{29}$.

Pour d'autres villes de l'Italie septentrionale et centrale, les sources ne permettent pas toujours une connaissance aussi approfondie de la structure sociale des meneurs de mouvements de révolte du genre de la Pataria comme c'est le cas à Milan grâce à une tradition documentaire particulièrement favorable ${ }^{30}$. Toujours est-il que les informations dont nous disposons donnent à penser que, là aussi, les meneurs occupaient un rang social élevé. Pour la ville d'Alba dans le Piémont, on connaît un certain Buzi qui en tant que fils de prêtre pouvait rallier à lui la suite de l'évêque ${ }^{31}$. À Crémone, déjà, les premiers mouvements de révolte urbaine contre la domination épiscopale en ville laissent entrevoir l'alliance entre la "puissance économique, l'union interne et un commandement politiquement expérimenté ${ }^{32}$. L'organisation de la Pataria de Crémone dans les années 1060 et 1070 se trouvait entre les mains d'un comité de douze hommes sous la direction d'un abbé du nom de Christophe. Son monastère (S. Pietro al Po) se trouvait dans un quartier avec beaucoup de monastères nouvellement fondés grâce au financement de Crémonais aisés, parmi lesquels des vassaux de l'évêque ${ }^{33}$. Après l'effondrement de la Pataria à Milan en 1075

${ }^{28}$ Voir André de Strumi, Vita Sancti Arialdi (voir n. 5) 6 (Nazarius), 11 (Azo), 20 (famille des Rozo) und 23 (Algisius), p. 1053, 1058, 1065, 1070.

${ }^{29}$ Voir à ce sujet GolnNell, La pataria (voir n. 18), p. 42-45; Marco NAVONI (éd.), Andrea da Strumi, Passione del santo martire milanese Arialdo. In appendice: Anonimo, Passione del beato Arialdo, sepolto nella chiesa di S. Dionigi. Introduzione, traduzione e cura, Milan 1994 (Biblioteca di Cultura Medievale, Storie e cronache), p. 25-26.

${ }^{30}$ Sur les problèmes de méthode pour la comparaison de la situation à Milan avec celle dans d'autres villes à cause des conditions différentes de la transmission des textes, voir KELLER, Pataria und Stadtverfassung (voir n. 18), p. 323, 327; ID., Mailand im 11. Jahrhundert (voir n. 19), p. 81-86; ZuMHAGEN, Religiöse Konflikte und kommunale Entwicklung (voir n. 13), p. 26-39.

${ }^{31}$ Voir Benzo von Alba, Ad Heinricum IV. imperatorem libri VII, IV 30 (1), éd. par Hans SEYFFERT, Hanovre 1996 (MGH SS rer. Germ., 65), p. 364-365; à ce sujet KELLER, Mailand im 11. Jahrhundert (voir n. 19), p. 94; ZUMHAGEN, Religiöse Konflikte und kommunale Entwicklung (voir n. 13), p. 130-135, surtout 132.

${ }^{32}$ Ibid., p. 145: Zusammenschluß von »ökonomischer Macht, Geschlossenheit und politisch erfahrener Führung«. Sur le conflit à Crémone entre 1027 et 1030, voir ibid., p. 142-147; KELLER, Der Übergang zur Kommune (voir n. 10), p. 59-60; François MENANT, Cremona in età precomunale: Il secolo XI, dans: Giancarlo ANDENNA (dir.), Storia di Cremona. Dall'alto medioevo all'età comunale, Crémone 2004, p. 106-197, ici p. 111.

${ }^{33}$ Voir Bonizon de Sutri, Liber ad amicum (voir n. 25) VI, p. 597; à ce sujet KELLER, Mailand im 11. Jahrhundert (voir n. 19), p. 97; ZUMHAGEN, Religiöse Konflikte und kommunale 
qui avait incité bon nombre de ses partisans à fuir vers Crémone, l'écrivain et spécialiste de droit canonique Bonizon de Sutri semble avoir lui aussi renforcé l'agitation en faveur de la Pataria à Crémone. D'après ses propres dires, la $\mathrm{Pa}$ taria fut soutenue par l'ensemble du peuple crémonais ${ }^{34}$. En 1088, Bonizon fut élevé par des partisans de la Pataria à l'évêché de Plaisance, mais il fut incapable de se maintenir face à la résistance des milites. Il échoua parce, en tant qu'étranger à cette région, tout lien avec la noblesse de Plaisance lui faisait défaut ${ }^{35}$. On désigne par le terme de $» P a t a r i a$ fiorentina « le mouvement de renouvellement des monastères de Vallombreuse, près de Florence ${ }^{36}$, inauguré par Jean Gualbert en $1036^{37}$. Son action d'agitateur dans les années 1060 contre l'évêque Pierre de Mezzabarba, considéré comme simoniaque, bénéficia du soutien de toute la commune urbaine et fut encouragée d'une manière efficace par les grands de Toscane ${ }^{38}$.

À eux seuls, ces quelques exemples montrent que le succès de révoltes urbaines dans l'Italie septentrionale et centrale dépendait essentiellement de l'ancrage de ses meneurs dans les classes sociales dirigeantes des villes et des

Entwicklung (voir n. 13), p. 153-154, 170; MENANT, Cremona in età precomunale (voir n. 32), p. 107, 127-132, également à propos de la structure sociale. Menant montre des parallélismes évidents avec la situation à Milan en renvoyant à la continuité entre les révoltes à Crémone motivées plutôt économiquement et socialement de la première moitié du $\mathrm{XI}^{\mathrm{e}}$ siècle et celles plutôt religieusement motivées de la deuxième moitié du $\mathrm{XI}^{\mathrm{e}}$ siècle.

${ }^{34}$ Voir Bonizon de Sutri, Liber ad amicum (voir n. 25) VII, p. 605; à ce sujet ZUMHAGEN, Religiöse Konflikte und kommunale Entwicklung (voir n. 13), p. 154; MENANT, Cremona in età precomunale (voir n. 32), p. 129.

${ }^{35}$ Voir Pierre RACINE, Plaisance du $X^{e}$ à la fin du XIII ${ }^{e}$ siècle. Essai d'histoire urbaine 1, Lille, Paris 1980, p. 215-224; ZUMHAGEN, Religiöse Konflikte und kommunale Entwicklung (voir n. 13), p. 163-169; Pierre RACINE, Capitanei à Plaisance, dans: Andrea CASTAGNETTI (dir.), La vassallità maggiore del regno Italico. I capitanei nei secoli XI-XII. Atti di convegno Verona, 4-6 novembre 1999, Rome 2001, p. 189-205.

${ }^{36}$ Paolo GolINELLI, I Vallombrosani e i movimenti patarinici, dans: Giordano Monzio COMPAGNONI (dir.), I Vallombrosani nella società italiana dei secoli XI e XII. Vallombrosa, 3-4 settembre 1993, Vallombrosa 1995 (Archivio Vallombrosano, 2), p. 35-56, ici p. 44; ZUMHAGEN, Religiöse Konflikte und kommunale Entwicklung (voir n. 13), p. 179.

${ }^{37}$ Voir Vita Iohannis Gualberti auctore Andrea abbate Strumensi, éd. par Friedrich BAETHGEN, dans: MGH SS 30, 2, Leipzig 1934, p. 1080-1104; Vita Iohannis Gualberti auctore discipulo eius anonymo, éd. par Friedrich BAETHGEN, dans: MGH SS 30, 2, Leipzig 1934, p. 1104-1110; Werner GOEZ, Reformpapsttum, Adel und monastische Emeuerung in der Toscana, dans: FLECKENSTEIN (dir.), Investiturstreit und Reichsverfassung (voir n. 18), p. 205-239, ici p. 229-233; ID., Johannes Gualberti, Abt von Vallombrosa, dans: ID., Lebensbilder aus dem Mittelalter. Die Zeit der Ottonen, Salier und Staufer, Darmstadt 1998, p. 139-149; Anna BENEVENUTI, San Giovanni Gualberto e Firenze, dans: CoMPaGNONI (dir.), I Vallombrosani nella società italiana (voir n. 36), p. 83-112.

${ }^{38}$ Voir GoEZ, Reformpapsttum, Adel und monastische Erneuerung (voir n. 37), p. 233-239; Nicolangelo D'ACUNTO, Lotte religiose a Firenze nel secolo XI: aspetti della rivolta contro il vescovo Pietro Mezzabarba, dans: Aevum 67 (1993), p. 279-312; GolinelLI, I Vallombrosani e i movimenti patarinici (voir n. 36), p. 44-56; ZUMHAGEN, Religiöse Konflikte und kommunale Entwicklung (voir n. 13), p. 178-202. 
régions. Il fallait un appui financier pour convaincre et emporter une large adhésion à propos de la nécessité de changements sociaux et religieux. Le chevalier milanais Erlembald disposait de cet appui et put régner dans la métropole milanaise pendant plusieurs années sans être sérieusement inquiété ${ }^{39}$. Ce n'est qu'au moment où il brisa le consensus avec ses partisans pour contrôler en tant que maître tous les domaines de la vie urbaine qu'un mouvement massif se déclencha, qui à son tour fut porté par tous les groupes sociaux sous la direction de la noblesse ${ }^{40}$.

La carrière d'Erlembald renvoie d'une manière générale aux qualités de ceux qui se sentaient appelés à mener un mouvement de révolte contre la domination des évêques et leur suite féodale dans les villes. Il est impossible, faute de sources provenant d'eux-mêmes, de tirer au clair leurs motivations. $\grave{A}$ côté de motifs sociaux et religieux incontestables, des intérêts économiques voire politiques très concrets auront sans doute joué leur rôle ${ }^{41}$. Pour gagner les communes urbaines à leurs intérêts particuliers, les meneurs des révoltes communales devaient surtout pouvoir se présenter de façon convaincante dans le public. Seul l'espace public pouvait fournir la possibilité de mobilisation d'un grand groupe de partisans pour faire vaciller ou même pour renverser les structures du pouvoir existantes ${ }^{42}$. Ses partisans comme ses opposants étaient d'accord sur les capacités magistrales de rhétorique dont disposait le protagoniste de la Pataria milanaise Landulf, de même nul ne contestait le caractère charismatique de la personnalité de Jean Gualbert de Vallombreuse ${ }^{43}$. Même si les sermons transmis sous le nom de Landulf sont les produits d'un remanie-

${ }^{39}$ Voir KELLER, Adelsherrschaft und städtische Gesellschaft (voir n. 5), p. 210-212 avec les références; ZUMHAGEN, Religiöse Konflikte und kommunale Entwicklung (voir n. 13), p. 57-64.

${ }^{40}$ Voir KELLER, Pataria und Stadtverfassung (voir n. 18), p. 325-326, 331, 343, 345-346; ZUMHAGEN, Religiöse Konflikte und kommunale Entwicklung (voir n. 13), p. 73-75, 89-93.

${ }^{41}$ Sur le problème du dégagement des motifs d'action de chacun des acteurs malgré la partialité des sources, voir déjà KELLER, Pataria und Stadtverfassung (voir n. 18), p. 330-333.

${ }^{42}$ Voir les réflexions de ZUMHAGEN, Religiöse Konflikte und kommunale Entwicklung (voir n. 13), p. 80-83 sur le sujet »le public comme forme de débat et d'agitation? « (Öffentlichkeit als Forum der Diskussion und Agitation?).

${ }^{43}$ Sur Landulf voir Arnulf de Milan, Liber gestorum recentium (voir n. 21) III 8, p. 175-176: Landulfus vero cum esset expeditioris lingue ac vocis nimiusque favoris amator, repente dux verbi efficitur usurpato sibi contra morem ecclesie predicationis officio; André de Strumi, Vita Sancti Arialdi (voir n. 5) 5, p. 1053: Landulfus, de urbanis excellentibus tam ordine quam natione, nimis potens in voce et sermone; Landulf l'Ancien, Historia Mediolanensis (voir n. 21) III 5, MGH SS 8, p. 76/RIS ${ }^{24}$, 2, p. 86: Landulfus de magna prosapia oriundus ... Itaque lingua eius modo dulcem, modo amaram aquam turpissimis verbis discurrentibus perfundens, nunc clanculo nuncque aperte omnes detrahens, quasi adamas ipsorum corda induraverat; Bonizon de Sutri, Liber ad amicum (voir n. 25) VI, p. 591: Landulfus ex maiorum prosapia ortus, vir urbanus et facundissimus. Sur Jean Gualbert voir les vies citées à la n. 37 et à propos de celles-ci ZUMHAGEN, Religiöse Konflikte und kommunale Entwicklung (voir n. 13), p. 182-184. 
ment littéraire, ils renvoient, tout comme les lettres de Jean Gualbert, au niveau culturel élevé de leurs auteurs ${ }^{44}$. Les différentes étapes de leur formation intellectuelle ne se distinguent que difficilement. On suppose qu'Ariald, partisan de la Pataria de Milan, avait acquis son savoir dans le domaine de la théologie lors de séjours d'étude à l'étranger ${ }^{45}$. De même les chevaliers dirigeants de tels mouvements devaient être en mesure de rallier la communauté urbaine derrière eux. Erlembald atteignit cet objectif d'une façon spectaculaire en faisant porter la dépouille d'Ariald assassiné en 1066 à travers la ville et en le faisant vénérer comme martyr ${ }^{46}$.

De toute façon, les meneurs ne devaient pas avoir peur de la mort. Les chefs de la Pataria milanaise Ariald, Landulf et Erlembald périrent tous d'une mort violente $^{47}$. Bonizon de Sutri fut mutilé au visage avant qu'on ne le chassât de Plaisance ${ }^{48}$. Il partagea cette destinée avec beaucoup d'autres ${ }^{49}$. Des peines

44 Des sermons de Landulf que l'on pourra considérer comme typiques de la façon d'argumenter des partisans de la Pataria sont transmis par Arnulf de Milan, Liber gestorum recentium (voir n. 21) III 9, p. 176-178, André de Strumi, Vita Sancti Arialdi (voir n. 5) 10, p. 1055-1057 et Landulf l'Ancien, Historia Mediolanensis (voir n. 21) III 25, MGH SS 8, p. 91-92/RIS 24, 2, p. 113-114 (Oratio Arialdi et Landulfi). Voir Giovanni MiCCOLI, Per la Storia della Pataria Milanese, dans: ID., Chiesa Gregoriana. Ricerche sulla Riforma del secolo XI, Florence 1966 (Storici antichi e moderni. Nuova serie, 17), p. 101-167, surtout 146149; KELLER, Pataria und Stadtverfassung (voir n. 18), p. 337; GolINELLI, La pataria (voir n. 18), p. 26. Deux lettres de Jean Gualbert ont été insérées par André de Strumi dans la Vita S. Iohannis Gualberti (voir n. 37) 67 et 80, p. 1093-1094, 1100-1101. L'agitation de Vallombrosa est relatée également par voie indirecte dans la lettre des Florentins à Alexandre II, ibid., 75, p. 1096-1099. À ce sujet Giovanni MICCOLI, Pietro Igneo. Studi sull' età gregoriana, Rome 1960 (Istituto storico Italiano per il medio evo. Studi storici, 40-41), p. 139-157. Voir par ailleurs Desiderius von Montecassino, Dialogi de miraculis Sancti Benedicti III 4, éd. par Gerhard SCHWARTZ, Adolf HOFMEISTER, dans: MGH SS 30, 2, Leipzig 1934, p. 1146-1147.

${ }^{45}$ Voir André de Strumi, Vita Sancti Arialdi (voir n. 5) 4, p. 1051 et les explications de NAVONI, Andrea da Strumi (voir n. 29), p. 59 n. 11.

46 Voir Andre de Strumi, Vita Sancti Arialdi (voir n. 5) 23-25, p. 1070-1072; Landulf l'Ancien, Historia Mediolanensis (voir n. 21) III 30, MGH SS 8, p. 95-96/RIS ${ }^{24}, 2$, p. 121-122.

${ }^{47}$ Voir le résumé de Golinelli, La pataria (voir n. 18), p. 35-41 ainsi que, plus en détail, Francesca ROVERSI MONACO, Landolfo, dans: Dizionario biografico degli Italiani 63 (2004), p. 486-489; Cosimo Damiano FONSECA, Arialdo, dans: Dizionario biografico degli Italiani 4 (1962), p. 135-139; Alfredo LUCIONI, Arialdo, santo ( $\dagger$ 1066), dans: Dizionario della chiesa ambrosiana 1 (1987), p. 254-255; ID., Erlembaldo, santo ( $\dagger$ 1075), dans: Dizionario della chiesa ambrosiana 2 (1988), p. 1129-1131; Jörg W. BUSCH, Hagen KELLER, Erlembaldo, dans: Dizionario biografico degli Italiani 43 (1993), p. 205-209.

48 Voir Giovanni MICCOLI, Bonizone, dans: Dizionario biografico degli Italiani 12 (1970), p. 246-259, ici p. 248.

${ }_{49}^{4}$ P.ex. le clerc Liprand/Liutprandus favorable à la Pataria gravement mutilé en relation avec l'assassinat d'Erlembald à Milan en 1075, voir Arnulf de Milan, Liber gestorum recentium (voir n. 21) IV 10, p. 216 et n. 92 sur les sources parallèles. ZUMHAGEN, Religiöse Konflikte und kommunale Entwicklung (voir n. 13), p. 169 renvoie à la mutilation des partisans de la Pataria comme symbole de la défaite de tout le mouvement. 
corporelles, la destruction des maisons et la mise en exil furent des peines typiques des communes qui étaient appliquées encore plus souvent en temps de révoltes urbaines ${ }^{50}$.

Dans ce contexte, la communauté des citadins apparaît plutôt comme une foule déchaînée que comme un organe de décision fondée sur le consensus. Pourtant, la capacité de s'unir et de renforcer cette union par un serment est attestée assez tôt à Milan. L'influence marquante du mouvement français de la paix de Dieu est patente ${ }^{51}$.

L'insurrection populaire milanaise contre la noblesse se termina en 1044 par une union de paix devant les portes de la ville et une messe en commun dans l'église du patron de la ville, saint Ambroise ${ }^{52}$. Depuis 1057 de grands rassemblements populaires sont attestés dans la ville ${ }^{53}$. Des cloches ou des trompettes servaient de signaux indiquant en même temps le lieu de rassemblement (le théâtre, la cathédrale ou Saint-Ambroise). D'abord les rassemblements permirent aux révoltés de faire connaître leurs doléances et de soumettre les groupes d'opposants à leur volonté, ce qui leur réussit grâce aux premières conjurations (coniurationes ou iuramenta communia) ${ }^{54}$. Les détenteurs tradi-

${ }^{50}$ Voir KELLER, Pataria und Stadtverfassung (voir n. 18), p. 339-340, surtout n. 55-56; ID., Der Übergang zur Kommune (voir n. 10), p. 65.

${ }^{51}$ Sur le débat autour de l'influence du mouvement de la paix de Dieu sur la formation des communes qu'approuve Hagen Keller contrairement à Hans-Werner Goetz et Knut Schulz voir KELLER, Mailand im 11. Jahrhundert (voir n. 19), p. 92-93 n. 58; ZUMHAGEN, Religiöse Konflikte und kommunale Entwicklung (voir n. 13), p. 204 n. 5.

${ }^{52}$ Voir Arnulf de Milan, Liber gestorum recentium (voir n. 21) II 19 et III 1, p. 165, 168169; Landulf l'Ancien, Historia Mediolanensis (voir n. 21) II 26, MGH SS 8, p. 65/RIS 24, 2 , p. 67; KELLER, Die soziale und politische Verfassung Mailands (voir n. 8), p. 51-52; ID., Pataria und Stadtverfassung (voir n. 18), p. 336-337; SCHULZ, "Denn sie lieben die Freiheit so sehr« (voir n. 1), p. 28; ZUMHAGEN, Religiöse Konflikte und kommunale Entwicklung (voir n. 13), p. 78-80.

${ }^{53}$ Voir, également pour ce qui suit, Landulf l'Ancien, Historia Mediolanensis (voir n. 21) III 9 , MGH SS 8, p. 80/RIS 24, 2, p. 92-93; à ce sujet KELLER, Die soziale und politische Verfassung Mailands (voir n. 8), p. 52-53; ID., Pataria und Stadtverfassung (voir n. 18), p. 337-340; ZUMHAGEN, Religiöse Konflikte und kommunale Entwicklung (voir n. 13), p. 82-93.

${ }^{54}$ Pour les iuramenta communia de 1057 et $1066 / 67$ provoqués par les partisans de la Pataria voir Amulf de Milan, Liber gestorum recentium (voir n. 21) III 11 et 18, p. 180, 194; à ce sujet KELLER, Die soziale und politische Verfassung Mailands (voir n. 8), p. 52; ID., Pataria und Stadtverfassung (voir n. 18), p. 338-339; ZUMHAGEN, Religiöse Konflikte und kommunale Entwicklung (voir n. 13), p. 93-95. Voir aussi les réactions au soulèvement des vavasseurs de 1035 hors de l'Italie du nord que relève KELLER, Pataria und Stadtverfassung (voir n. 18), p. 333. Sur les coniurationes voir en général Otto Gerhard OEXLE, Die Kultur der Rebellion. Schwureinung und Verschwörung im früh- und hochmittelalterlichen Okzident, dans: Marie Theres FOGEN (dir.), Ordnung und Aufruhr im Mittelalter. Historische und juristische Studien zur Rebellion, Francfort/M. 1995 (Ius commune: Sonderhefte. Studien zur europäischen Rechtsgeschichte, 70), p. 119-137; ID., Friede durch Verschwörung, in: Johannes FRIED (dir.), Träger und Instrumentarien des Friedens im hohen und späten Mittelalter, Sigmaringen 1996 (Vorträge und Forschungen, 43), p. 115-150, surtout p. 121-123 sur les mouvements communaux en Italie et en France. 
tionnels du pouvoir en revanche apprirent vite ${ }^{55}$ et se servirent à leur tour du rassemblement populaire comme moyen d'imposer leurs décisions. Si l'on en croit les récits de contemporains milanais, il était tout à fait normal de délibérer dans les rues et sur les places publiques sur des questions religieuses ${ }^{56}$. Le rassemblement populaire était ainsi devenu partie intégrante de la vie politique milanaise ${ }^{57}$. Déjà depuis la fin des années 1060 , des conjurations s'étaient formées sur la base d'un consensus de plus en plus large de tous les groupes de la population ${ }^{58}$. La commune urbaine tira de cette mise en œuvre commune pour la paix en ville un sentiment de sa propre valeur que personne ne pouvait désormais négliger. Même après la fin de l'agitation de la Pataria, seul celui qui se savait en accord avec les représentants de l'assemblée populaire était capable de gouverner dans la métropole lombarde ${ }^{59}$. Déjà vers 1080 , il y a des indices concrets de l'existence de représentants élus de l'assemblée populaire ${ }^{60}$ qui s'intensifient vers le tournant du siècle ${ }^{61}$. A partir de 1120 il n'y a plus de doute possible. Un conseil urbain se forme à partir de l'assemblée populaire; il est constitué de sept capitanei, sept vavasseurs et six personnalités estimées de $\mathrm{Milan}^{62}$. La première mention des vingt consuls de Milan date de l'an $1117^{63}$.

Pour Crémone, Plaisance et Florence aussi, on connaît l'importance de l'assemblée populaire en tant qu'embryon des institutions communales ${ }^{64}$.

${ }^{\text {s5 }}$ Voir ZUMHAGEN, Religiöse Konflikte und kommunale Gesellschaft (voir n. 13), p. 95, 205.

${ }^{56}$ Voir ibid., surtout p. 204.

${ }^{57}$ Voir KELLER, Die soziale und politische Verfassung Mailands (voir n. 8), p. 52-55; ID., Pataria und Stadtverfassung (voir n. 18), p. 337-338, n. 45, 47, p. 340.

${ }^{58}$ Sur la conjuration de 1067 voir les sources mentionnées à la note 20 ainsi que Arnulf de Milan, Liber gestorum recentium (voir n. 21) Шl 21, p. 195-196; pour la conjuration de 1071 a l'occasion de l'élection d'un nouvel archevêque voir ibid., III 23, p. 203 ainsi que pour la conjuration de 1075 menant à la chute d'Erlembald ibid., IV 10-11, p. 215-218; Landulf l'Ancien, Historia Mediolanensis (voir n. 21) $\amalg$ 30, MGH SS 8, p. 96/RIS 24, 2, p. 122; Bonizon de Sutri, Liber ad amicum (voir n. 25) VI, p. 602, 604-605; à ce sujet KELLER, Die soziale und politische Verfassung Mailands (voir n. 8), p. 51-52; ID., Pataria und Stadtverfassung (voir n. 18), p. 331, 337, 344-346; ZUMHAGEN, Religiöse Konflikte und kommunale Entwicklung (voir n. 13), p. 87-88.

${ }^{59}$ Voir pour ce qui suit ibid., p. 98-128.

${ }^{60}$ Voir ibid., p. 107.

${ }^{61}$ Pour la soi-disant première mention de consuls milanais en 1097 voir DILCHER, Die Entstehung der lombardischen Stadtkommune (voir n. 11), p. 131-132; ZUMHAGEN, Religiöse Konflikte und kommunale Entwicklung (voir n. 13), p. 117.

${ }^{62}$ Voir SCHULZ, wDenn sie lieben die Freiheit so sehr" (voir n. 1), p. 47.

${ }^{63}$ Voir ibid.; DILCHER, Die Entstehung der lombardischen Stadtkommune (voir n. 11), p. 133-134; ZUMHAGEN, Religiöse Konflikte und kommunale Entwicklung (voir n. 13), p. 125128. L'existence ininterrompue d'une constitution basée sur des consuls ne peut cependant être prouvée qu'après 1138 selon KELLER, Mailand im 11. Jahrhundert (voir n. 19), p. 97.

${ }^{64}$ Pour Milan, Crémone et Plaisance voir DilchER, Die Entstehung der lombardischen Stadtkommune (voir n. 11), p. 139-140; à ce sujet dans une perspective critique ZUMHAGEN, Religiöse Konflikte und kommunale Entwicklung (voir n. 13), p. 169-177; pour Florence ibid., p. 195-202. 
Seule l'assemblée florentine peut néanmoins être mise en relation évidente avec le mouvement contestataire religieux de Vallombreuse.

Les moyens par lesquels d'autres villes de l'Italie septentrionale et centrale, de cités auparavant dirigées par des évêques ou margraves qu'elles étaient, se transformèrent en communes restent pour la plupart obscurs ${ }^{65}$. Or des privilèges royaux des années 1080 pour les habitants de Lucques, Pise, Mantoue et Plaisance montrent que les structures établies du pouvoir connurent aussi à d'autres endroits des crises dans le sillon de la réforme de l'Église et de la querelle des investitures ${ }^{66}$. Les habitants font face au roi en tant que communauté consciente d'elle-même et se font rémunérer leur fidélité au roi par l'attribution de larges droits. On leur garantit que leurs villes et leur statut juridique demeureraient indemnes, on les libère de redevances et de services, on encourage le commerce et la circulation. Il restait, également pour ces villes-là, un bon bout de chemin jusqu'à la formation d'une constitution communale, mais l'aspiration à l'autonomie juridique et économique se distingue déjà nettement.

La situation à Milan montre d'une façon particulière à quel point notre connaissance du développement des communes dépend de la transmission de textes historiographiques ${ }^{67}$. Aucune autre ville ne présente une transmission aussi dense de savoir historique pour le $\mathrm{XI}^{\mathrm{e}}$ et $\mathrm{XII}^{\mathrm{e}}$ siècle, qui nous renseigne sur la perception et l'appréciation des débuts du mouvement communal et de leurs meneurs. L'intérêt particulier de ces écrits réside dans le parti pris résolu des auteurs. Il s'agit d'écrits polémiques dans le meilleur sens du terme, qui devaient servir aussi bien à réconforter les partisans qu'à dénoncer le parti adverse. Les personnages prépondérants dans l'action et dans l'agitation étaient au centre de l'intérêt. Or les louanges et la critique étaient exprimées dans des formes typologiquement différentes, ce qui renvoie à un autre mode de perception. Les propos panégyriques étaient exprimés ou bien dans des vies de saints comme dans les vies d'Ariald et de Jean Gualbert par l'abbé André de Stru$\mathrm{mi}^{68}$, ou bien ils se trouvaient dans des passages hagiographiques insérés dans le cours du récit comme chez Bonizon de Sutri ${ }^{69}$. L'ardeur religieuse des protagonistes est particulièrement mise en relief. Ils sont présentés comme mar-

\footnotetext{
${ }^{65}$ Voir l'examen de la bibliographie chez KELLER, Mailand im 11. Jahrhundert (voir n. 19), passim dans les notes.

${ }^{66}$ Sur les actes de Henri IV, éd. par Dietrich voN GLADISS, Alfred GAWLIK (MGH Diplomata regum et imperatorum, 6), Berlin, Weimar, Hanovre 1941-1978, ${ }^{\circ} 334,336,421, * 519$ voir Tilman STRUVE, Heinrich IV. und die fideles cives der städtischen Kommunen Oberitaliens, dans: Deutsches Archiv für Erforschung des Mittelalters 53 (1997), p. 497-553, aussi pour ce qui suit.

${ }^{67}$ Pour ce qui suit, voir aussi n. $25,30$.

${ }^{68}$ Voir n. 5, 37.

${ }^{69}$ Voir Bonizon de Sutri, Liber ad amicum (voir n. 25) VI, p. 596-597 sur Ariald ainsi que VI, p. 598-599 et VII, p. $604-605$ sur Erlembald.
} 
tyrs de la vraie foi. L'impact de leur action sur des changements au sein de la société n'est guère évoqué.

Il en allait tout autrement dans les æuvres historiographiques de leurs adversaires. Ces derniers prirent la plume avec l'objectif unique de dénoncer le fait que l'ordre social considéré par eux comme approprié avait été sérieusement ébranlé par ces soulèvements. Le changement du status urbis et ecclesie $e^{70}$, donc de l'état de la ville et de l'Église, constitue pour eux l'origine de tous les maux $^{71}$. La critique du comportement de ceux qui transgressent leur condition sociale dans un objectif révolutionnaire n'en ressort que plus violente. Le chevalier Lanzo est critiqué pour avoir dirigé un mouvement populaire en tant que représentant d'une famille de la noblesse ${ }^{72}$. On blâme le chevalier Erlembald de s'être mêlé, en tant que laïc, des affaires ecclésiastiques ${ }^{73}$. On s'attaque au clerc Landulf parce qu'il s'était arrogé le droit de prêcher sans avoir été ordonné prêtre ${ }^{74}$.

Il ne s'agit pas que du wrefus conscient ou de la diabolisation du nouveau en tant que nouveauté ${ }^{75}$, mais au contraire du dédain ostentatoire des limites de la condition sociale et de l'incitation d'autrui au mépris de ces limites. On décrit les conséquences dans des termes évoquant la confusion et le chaos ${ }^{76}$. Le chroniqueur Benzon d'Alba n'hésita pas à s'adresser directement au roi et aux évêques de l'Italie du nord en leur demandant de mettre un terme à cette folie. Il s'écria vers l'archevêque de Milan: »Ne tournez pas le dos aux lâches, mon-

${ }^{70}$ Arnulf de Milan, Liber gestorum recentium (voir n. 21) II 18 et III Prologue, p. 163, 167.

${ }^{11}$ Voir KELLER, Mailand im 11. Jahrhundert (voir n. 19), p. 95-97; ZUMHAGEN, Religiöse Konflikte und kommunale Entwicklung (voir n. 13), p. 28-34 avec la bibliographie ancienne.

${ }^{72}$ Voir Arnulf de Milan, Liber gestorum recentium (voir n. 21) II 18, p. 163-164 (avec le relevé des sources parallèles dans les notes): Lanzo quidam ingenuus civitatis miles plebeie turbe favebat instantius, cuius fota iuvamine plurimum convalescebat.

${ }^{73}$ Voir Arnulf de Milan, Liber gestorum recentium (voir n. 21) III 14, p. 187 (avec le relevé des sources parallèles dans les notes): Arialdus itaque tali destitutus collega instigat Arlembaldum assidue defuncti fratris vicem suscipere. Qui cum esset laycus, quasi fraterne gratia pietatis opus sibi presumpsit indebitum... Dum ergo laicus iudicat, clericus tantum vapulat.

${ }^{74}$ Voir Arnulf de Milan, Liber gestorum recentium (voir n. 21) III 8, p. 175-176 (avec le relevé des sources paralleles dans les notes): Landulfus vero cum esset expeditioris lingue ac vocis nimiusque favoris amator, repente dux verbi efficitur usurpato sibi contra morem ecclesie predicationis officio.

${ }^{75}$ Knut SCHULZ, Die Reaktion auf die frühe kommunale Bewegung vom Ende des 11. bis zur Mitte des 12. Jahrhunderts, dans: Hans-Joachim SCHMIDT (dir.), Tradition, Innovation, Invention. Fortschrittsverweigerung und Fortschrittsbewusstsein im Mittelalter, Berlin, New York 2005 (Scrinium Friburgense. Veröffentlichungen des Mediävistischen Instituts der Universität Freiburg Schweiz, 18), p. 335-360, ici p. 337: "bewußte Ablehnung oder Verteufelung des Neuen als Neues«.

${ }^{76}$ Voir Arnulf de Milan, Liber gestorum recentium (voir n. 21) IV 11-12, p. 217-219. 
trez votre face; rangez l'ordre terrible de la bataille contre ces gens; ceux qui se croient gros se verront décharnés « ${ }^{77}$.

L'analyse des événements et l'appréciation des personnages par les auteurs hostiles à la Pataria du XI ${ }^{e}$ siècle étaient marquées par la passion. Un autre niveau d'explication était réservé à des chroniqueurs disposant de plus de distance chronologique, personnelle et géographique. Otton de Freising, spécialement, en fait partie. Chez lui aussi, le refus de la nouveauté constitue une trame principale du récit, mais il s'aperçoit clairement de l'efficacité des communes sans être aveuglé par une haine partiale ${ }^{78}$. De même, d'autres observateurs de condition ecclésiastique commentaient les mouvements communaux dans d'autres régions de l'Europe dans une perspective semblable ${ }^{79}$.

Il est révélateur que les communes ne trouvaient du soutien que chez des auteurs de condition laïque ${ }^{80}$. Revenons une dernière fois à Milan où l'histoire était apparemment écrite uniquement par des laïcs du milieu du XII ${ }^{e}$ jusqu'au milieu du $\mathrm{XIII}^{\mathrm{e}}$ siècle $^{81}$. Parmi eux se trouvaient même quelques notaires qui participaient à la vie de la commune par des décisions gouvernementales et en tant que détenteurs d'offices. Ils se proposaient comme sujet l'ambition de la commune des habitants vers l'autonomie et les débuts d'un nouvel ordre social. Contrairement à leurs prédécesseurs ecclésiastiques, ils choisissaient comme fil directeur le développement juridique et prenaient par là une nouvelle perspective conformément à la nouvelle époque qui s'annonçait ${ }^{82}$.

Dans leurs œuvres se reflète néanmoins aussi un changement de paradigme pour l'histoire des communes italiennes dans la deuxième moitié du XII ${ }^{e}$ siècle. Avec l'entrée en scène de Frédéric Barberousse en Italie, les communes se

${ }^{77}$ Benzon d'Alba, Ad Heinricum IV Imperatorum (voir n. 31) IV 30, p. 358-359: Non ignavis terga dentur, demonstrate faciem; Ordinate contra tales terribilem aciem; Qui videntur sibi pingues, vertantur in maciem.

${ }^{78}$ Voir $\mathrm{n} .7$.

${ }^{79}$ SCHULZ, Die Reaktion auf die frühe kommunale Bewegung (voir n. 75), p. 337 divise les réactions aux débuts du mouvement communal en quatre catégories, dont il décrit la deuxième comme »le refus dans une perspective néanmoins attentive et respectueuse envers le caractère innovatif et ses répercussions« (»Die Zurückweisung mit einem aufmerksamen oder respektvollen Blick auf das Innovative und seine Auswirkungen (). Il mentionne ibid., p. 346-347, à côté d'Otton de Freising l'attitude respectueuse face au soulèvement des habitants de Worms chez Lampert de Hersfeld et les nuances qui apparaissent dans le récit de Guibert de Nogent malgré son rejet général des mouvements communaux à Laon.

${ }^{80}$ SCHULZ, Die Reaktion auf die frühe kommunale Bewegung (voir n. 75), p. 348-353 mentionne sous sa troisième catégorie wl'acceptation ou l'encouragement intentionnels du nouveau en tant que nouveauté ("Die bewußte Bejahung oder Förderung des Neuen als Neues $\ll$ ) trois actes royaux.

${ }^{81}$ Voir la vue d'ensemble chez BUSCH, Die Mailänder Geschichtsschreibung (voir n. 16), p. 35; à ce sujet Claudia ZEY, Zu Editionen und Interpretationen von Mailänder Geschichtswerken des Hoch- und Spätmittelalters, dans: Deutsches Archiv für Erforschung des Mittelalters 56 (2000), p. 179-199, ici p. 195-197.

${ }^{82}$ Voir BuSCH, Die Mailänder Geschichtsschreibung (voir n. 16), p. 70-144, 234-243. 
virent confrontées à des prétentions à la domination par déduction du droit romain et d'une grande portée, puisque celles-ci déniaient en fait l'autonomie urbaine. La réponse des communes à cette pression extérieure fut, comme on le sait, la fédération organisée dans la ligue lombarde à laquelle même Barberousse dut finalement se plier ${ }^{83}$.

${ }^{83}$ Voir à ce sujet le passage pertinent chez KELLER, Pataria und Stadtverfassung (voir n. 18), p. 321 avec la bibliographie plus ancienne; Alfred HAVERKAMP, Die Städte im Herrschaftsund Sozialgefuige Reichsitaliens, dans: Friedrich VITTINGHOFF (dir.), Stadt und Herrschaft. Römische Kaiserzeit und Hohes Mittelalter, Munich 1982 (Historische Zeitschrif, Beiheft N.F. 7), p. 149-245; Renato BORDONE, I communi italiani nella prima Lega Lombarda: confronto di modelli istituzionali in un'esperienza politico-diplomatica, dans: Helmut MAURER (dir.), Kommunale Bündnisse Oberitaliens und Oberdeutschlands im Vergleich, Sigmaringen 1987 (Vorträge und Forschungen, 33), p. 45-61; SCHULZ, »Denn sie lieben die Freiheit so sehr« (voir n. 1), p. 187-216. 
